\title{
Développement d'un protocole de suivi et conservation des épaves
}

\section{Monitoring protocol for the preservation of ancient wooden shipwrecks}

\author{
Marie-Laure Courboulès ${ }^{1}$, Daniela Peloso ${ }^{2}$, Vincent Dumas ${ }^{3}$ \\ ${ }^{1}$ Musée départemental Arles antique, Conseil départemental des Bouches-du-Rhône, laure.courboules@cg13.fr \\ ${ }^{2}$ Ipso Facto, Bureau d'étude et de recherche en archéologie subaquatique et sous-marine, Marseille, \\ daniela.peloso@ipsofacto.com \\ ${ }^{3}$ Aix Marseille Univ, CNRS, MCC, CCJ, Centre Camille Jullian, F-13000, Aix-en-Provence, vdumas@mmsh.univ-aix.fr
}

RÉSUMÉ. Ce projet de suivi d'une épave s'inscrit dans le programme de conservation et restauration du Musée d'Arles Antique, qui, à partir de l'année 2014, en charge des problématiques inhérentes à la conservation d'un chaland de 31 mètres de longueur. L'étude de l'état de conservation et des déformations du chaland Arles-Rhône3 (AR3) a mis en évidence la nécessité de mettre en place un dispositif spécifique de suivi. L'absence d'un protocole d'étude nous a orientés vers un outil qui puisse être applicable par tous les conservateurs des musées, et par les archéologues qui ont identifié ce besoin.

Les épaves sont essentiellement composées de matériaux organiques (bois, calfatage), et très souvent munies d'assemblages impliquant des parties métalliques. II s'agit donc d'objets muséographiques composites et de taille souvent imposante. Ils sont cependant préférentiellement exposés directement dans les salles des musées, et, par conséquent, soumis aux variations climatiques. Force est de constater que la tendance consiste à accorder une trop grande confiance aux traitements de conservation/restauration initiaux, et à ne pas considérer l'évolution des objets après traitement. II est cependant important de considérer les objets dans leur contexte de préservation, et de mettre en évidence l'importance de la conservation préventive. Concernant la forme que pourra prendre ce protocole de suivi, plusieurs aspects de la prise en compte de l'espace et du temps doivent être évoqués. Les acteurs, issus de disciplines différentes, interviendront dans la définition d'un modèle théorique général de protocole, qui pourra faire émerger la notion de monitoring pour la conservation.

ABSTRACT. The experimental project of the preservation monitoring of a wreck is part of the program of preservation and restoration of the Departmental Museum of Ancient Arles. From 2014, the museum has dealt with the problems inherent to the preservation of a Roman barge 31 meters long exposed in its galleries. The study of the preservation status and of the deformations of the Roman maritime-fluvial barge Arles-Rhône3 (AR3) demonstrated the scientific necessity of an adapted monitoring tool, as well as of a monitoring protocol, applicable by the restorer/curator's team of a maritime Museum.

Ancient wooden shipwrecks are mainly composed of organic materials (wood, caulking, etc.), and frequently equipped with the metallic components (...). They constitute rare archaeological objects and are frequently of very big size. When exhibited, they are preserved in the halls of museums, and are, consequently, very sensitive to room climate variations.

Too often, there is a tendency to give too much attention to the initial restoration, and not consider enough the evolution of objects during the preservation process. Therefore, it is very important to consider the objects in their context and make the most of the preventive conservation. Concerning the content of the proposed monitoring protocol, several aspects of space and time must be considered. Moreover, the team responsible, consisting from scientists from different disciplines, will have to establish the general theoretical framework of the protocol for preservation monitoring.

MOTS-CLÉS. épave, conservation, suivi, photogrammétrie, SIG, base des données.

KEYWORDS. wreck, monitoring, preservation, photogrammetry, GIS, database platform.

\section{De la découverte à la fouille-relevage}

Le chaland AR3 a été découvert en 2004 dans le port romain d'Arelate (l'Arles romaine), sur la rive droite du Rhône, déposé sur la pente naturelle du fleuve. Cette épave est datée du milieu du $\mathrm{I}^{\text {er }}$ siècle après J.C. Suite à différentes campagnes d'expertise et de sondage, l'épave a fait l'objet de trois campagnes de fouille programmées de 2008 à 2010 (soit une totalité de trois mois et demie de travail). L'étude de l'architecture du bateau, associée à un important travail de dendrochronologie, a été conduite dans son 
contexte historique et environnemental. C'est en raison de son intérêt scientifique et patrimonial que le Conseil général des Bouches du Rhône, en accord avec le Département d'Archéologie Subaquatique et Sous-Marine (DRASSM), a décidé de compléter les fouilles et de sortir l'épave de l'eau. Durant cette fouille des moyens technologiques différents ont été utilisés. Une documentation systématique a été réalisée, que ce soit pour documenter la fouille stratigraphique du dépôt portuaire, des niveaux directement liés à l'épave, également pour tout ce qui concernait l'architecture même du chaland. Le relevage a été conçu à l'aide d'un châssis métallique, un "berceau" en forme de U, spécifiquement conçu pour amener les scaphandriers juste au-dessus du tronçon découpé, afin d'immobiliser chaque tronçon (pour une totalité de 10 tronçons) et de le récupérer facilement en totale sécurité. Une fois acheminés dans le hangar, les tronçons de l'épave ont été nettoyés par les archéologues et les restaurateurs, et ensuite, documentés, tout en étant humidifiés de façon continue. Simultanément, une véritable chaîne de conservation a été mise en place afin "d'assurer le suivi des objets" au fur et à mesure de leur sortie du fleuve. Par la suite, chaque détail et élément architectural des tronçons a fait l'objet d'un relevé $3 \mathrm{D}^{1}$ à partir de données obtenues via un outil de mesure tridimensionnelle, ceci dans l'objectif de produire une documentation précise. Enfin, les pièces de bois de l'épave, démontés et conditionnés, ainsi que toutes les parties métalliques, ont été restaurées avec des traitements spécifiques. Des échantillons de bois ont également été prélevés afin de réaliser des analyses xylologiques pour déterminer quelles ont été les essences de bois utilisées pour la construction du bateau.

Ces opérations de l'épave AR32 ${ }^{2}$, conduite en 2011, s'inscrivaient dans un projet plus complexe qui envisageait la restauration du chaland et son intégration dans le Musée d'Arles Antique, ce qui donné suite à la construction d'une extension du musée dédiée à cette épave (figure 1).

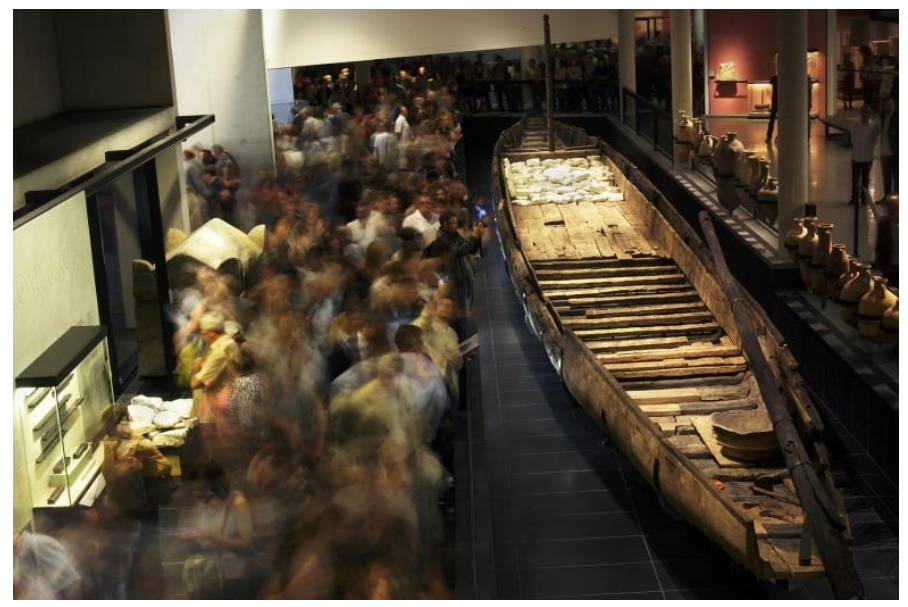

Figure 1. Le chaland Arles-Rhône 3 dans son installation muséale. Crédit : @ Rémi BENALI-MdAa-CD13

De plus, ce projet faisait partie de la programmation de "Marseille-Provence Capitale Européenne de la Culture 2013". En outre, le ministère de la Culture a classé en novembre 2010 le chaland Arles-Rhône 3 comme "trésor national".

\subsection{Traitement de conservation et de présentation}

La question de relever et de traiter le bateau en une seule pièce s'est posée dès le début du projet. Mais bien qu'idéale, cette solution a été rejetée pour plusieurs raisons. La première est liée au milieu fluvial.

1 Le relevé 3D a été réalisé à l'aide du C-Track, un outil de mesure conçu et commercialisé par la compagnie Creaform 3D

2 Maîtrise d'ouvrage : Conseil général des Bouches du Rhône; Maîtrise d'œuvre : Musée départemental Arles antique ; Responsable d'opération : Sabrina Marlier ; Opérateurs pour la fouille-relevage : groupement O'Can et Ipso Facto ; Opérateurs pour les traitements des bois et métaux : ARC-Nucleart et A-Corros ; Suivi scientifique : DRASSM 
Dégagé progressivement au cours de la fouille, le bateau se serait retrouvé fragilisé face aux caprices du fleuve. Le relevage, la manipulation et le transport d'une telle pièce, aussi fragile et aussi volumineuse, étaient impossibles et déraisonnables. Les infrastructures associées aux traitements proposés par l'atelier Arc Nucléart, chargé des traitements des bois, n'étaient pas non plus adaptées à une telle taille d'épave. Par ailleurs, construire un nouveau dispositif était inenvisageable dans les délais et dans le budget fixés. La solution de sectionner le bateau en plusieurs tronçons s'est imposée d'elle-même, et a été pensée pour tout le processus de fouille, de relevage et de conservation. Le bateau fut coupé en 10 sections de $3 \mathrm{~m}$ de long, et toutes ont été traitées (nettoyage, relevé....) séparément. A l'exception de la section avant du bateau, les autres furent démontés en plusieurs parties (désassemblage des flans et découpe de la sole en deux). Les pièces ainsi isolées ont été glissées sur des plateaux en inox et conditionnées pour être acheminées à Grenoble. Ces mêmes plateaux ont servi durant les quelques mois nécessaires à la procédure de traitement de conservation des bois gorgés d'eau. Ces opérations ont consisté à soumettre les éléments ligneux à différents bains d'imprégnation d'une résine (PEG, polyéthyléne glycol), qui a remplacé progressivement l'eau contenue dans les bois, puis à effectuer un séchage par lyophilisation. Le traitement complet des bois a duré deux ans.

Pour des raisons de prévention, un maximum de clous d'assemblage a été retiré avant traitement. En effet, les bains d'imprégnations durent plusieurs mois et provoquent l'oxydation des métaux, et le polyéthylène glycol utilisé pour imprégner les bois est un matériau hydrophile sensible à l'humidité ambiante. Cette propriété constitue un risque d'oxydation des sulfures de fer contenus dans le bois, qui se transforment en acide sulfurique dégradant potentiellement les bois. Ce risque d'acidification est commun à toutes les épaves -associant bois/fer- traitées au PEG dans les cinquante dernières années. Pour cette raison, la partie avant du bateau qui concentre une grande quantité de renforts métalliques a subi un traitement différent utilisant une résine radio durcissable, le styrène polyester (procédé Nucléart). Cette résine est moins sensible à l'humidité ambiante limitant ainsi le risque d'oxydation.

Suite au séchage par lyophilisation, de longues interventions de nettoyage, de consolidation et de remontage monopolisant plusieurs spécialistes ont été nécessaires, pour restaurer les bois, les parties métalliques et fabriquer le support. Au final, le bateau a été assemblé à nouveau sur un socle métallique de 8 tonnes.

Par choix muséographique, la partie manquante à l'arrière du bateau a été restituée en bois moderne, le mât calé dans son emplanture et maintenu vertical par des câbles, et la pelle de gouverne présentée sur son propre socle, en situation de navigation. Le bateau a été installé dans une fosse (figure 1) et apparaît aux visiteurs du musée tel qu'il devait être avant son naufrage, à quai, avec son matériel de bord et sa cargaison de pierres (fac similé en résine).

\section{La surveillance du bateau au musée}

Constitué de matériaux hygroscopiques, le bateau est sensible aux variations climatiques dans son lieu d'exposition. Suivant l'hygrométrie, les bois subissent des variations dimensionnelles qui peuvent entraîner des fendillements, des cassures, des soulèvements, des déformations... Le contrôle du climat (température et hygrométrie) est un des points clés de la conservation de ces collections.

Quelques mois après son installation dans les salles du musée, les espaces entre les sections se sont ouverts. Ces déformations ont fait l'objet d'un suivi par les équipes du musée (la conservation et les restaurateurs), en concertation avec l'équipe de l'atelier Arc'Nucléart. Cependant, les moyens de mesures ne permettent pas encore à ce jour de déterminer l'orientation spatiale de ces déformations. La question de leur évolution, et l'influence du climat sur ces dernières, est toujours une préoccupation majeure. 
En dehors du climat de conservation, la poussière est un facteur de dégradation non négligeable, car celle-ci provoque l'oxydation des métaux en fixant l'humidité, et favorise aussi le développement de micro-organismes qui peuvent attaquer les bois. Le calendrier de dépoussiérage a été établi d'après les observations réalisées pendant les contrôles réguliers effectués sur la barge.

Un constat d'état est programmé tous les ans, avec l'intervention de plusieurs spécialistes au côté des équipes du musée. Ces constats sont l'occasion d'établir un bilan sanitaire qui permet, à partir des informations recueillies, de suivre l'état du bateau dans le temps.

Face à ces observations et au nombre de données croissantes, se pose le problème du protocole d'enregistrement de ces différents paramètres et de leurs corrélations. Des liens entre climatéclairage/altérations-déformations, des liens entre publics/climat restent encore à établir.

Une des difficultés est l'enregistrement et la localisation précise des données en l'absence, d'un outil de représentation du bateau dans sa configuration actuelle, socle compris. De plus, les données enregistrées et les mesures réalisées étant de plus en plus nombreuses, la question de l'exploitation de ces données se pose également. Comment localiser, enregistrer et confronter toutes ces données ? En 2015 le musée s'est appuyé sur l'expertise technologique du bureau d'étude Ipso Facto pour répondre à ce besoin.

\section{Le développement du protocole}

Comme évoqué précédemment, l'étude de l'état de conservation et des déformations du chaland AR3 a mis en évidence la nécessité scientifique d'un dispositif adapté de suivi. Il doit permettre d'établir une corrélation chiffrée et cartographiée des interactions bateau-musée, aujourd'hui empiriquement constatées. L'absence d'un protocole d'étude nous a poussés à développer un outil complet, qui puisse être utilisé par toutes les équipes (conservateurs, archéologues) qui en auraient l'usage ${ }^{3}$.

La définition d'un suivi qui soit pertinent peut s'exprimer en trois niveaux complémentaires, propres à la réflexion archéologique : l'étude de l'objet, l'étude de son milieu et la prise en compte de leurs évolutions conjointes et réciproques dans le temps.

Le suivi doit donc associer la dimension temporelle à l'approche spatiale de la conservation préventive. Par ailleurs, et à l'échelle humaine, la continuité de ce suivi, requise pour une conservation pérenne des objets du patrimoine, n'est pas évidente à maintenir. En ce qui concerne les épaves, en étudiant les expériences de conservation de différents instituts, nous constatons que chaque organisme adopte en interne un mode de suivi propre. Les méthodes adoptées n'ont généralement pas pour objectif d'être adaptées à la spécificité des objets conservés que sont les épaves. Le plus souvent, elles ne considèrent pas l'évolution de la conservation par rapport aux conditions de préservation (directement liées à l'environnement de conservation). De plus, compte tenu des longues périodes sur lesquelles portent ces conservations, les modalités de suivi adoptées au fil des ans ne respectent pas toujours-les préconisations mentionnées par les équipes qui ont initié les restaurations. Or, ces préconisations sont des critères majeurs. Il est évident que la mémoire et la compréhension des phénomènes initiaux qui régissent une bonne conservation sont incontournables pour la détermination des paramètres de préservation, l'établissement d'un calendrier fiable des opérations à réaliser, et, d'une manière générale, la mise en place d'un protocole de suivi efficace.

3 Nous avons seulement pu confronter notre projet aux comptes rendus de différents projets de conservation qui, il faut le souligner, ne mentionnent exclusivement que des relevés numériques à une date donnée. Il s'agit des conservations de l'épave du Cog de Brème (Allemagne, projet COSCH), des épaves du Latenium et d'Yverdon (Suisse) et du Vasa (Stockholm, Suède). 
Le protocole repose sur un relevé topographique de contrôle de chaque élément architectural du chaland, sur un relevé photogrammétrique de la totalité de l'objet et sur la mise en relation des informations numériques avec une base de données (figure 2).

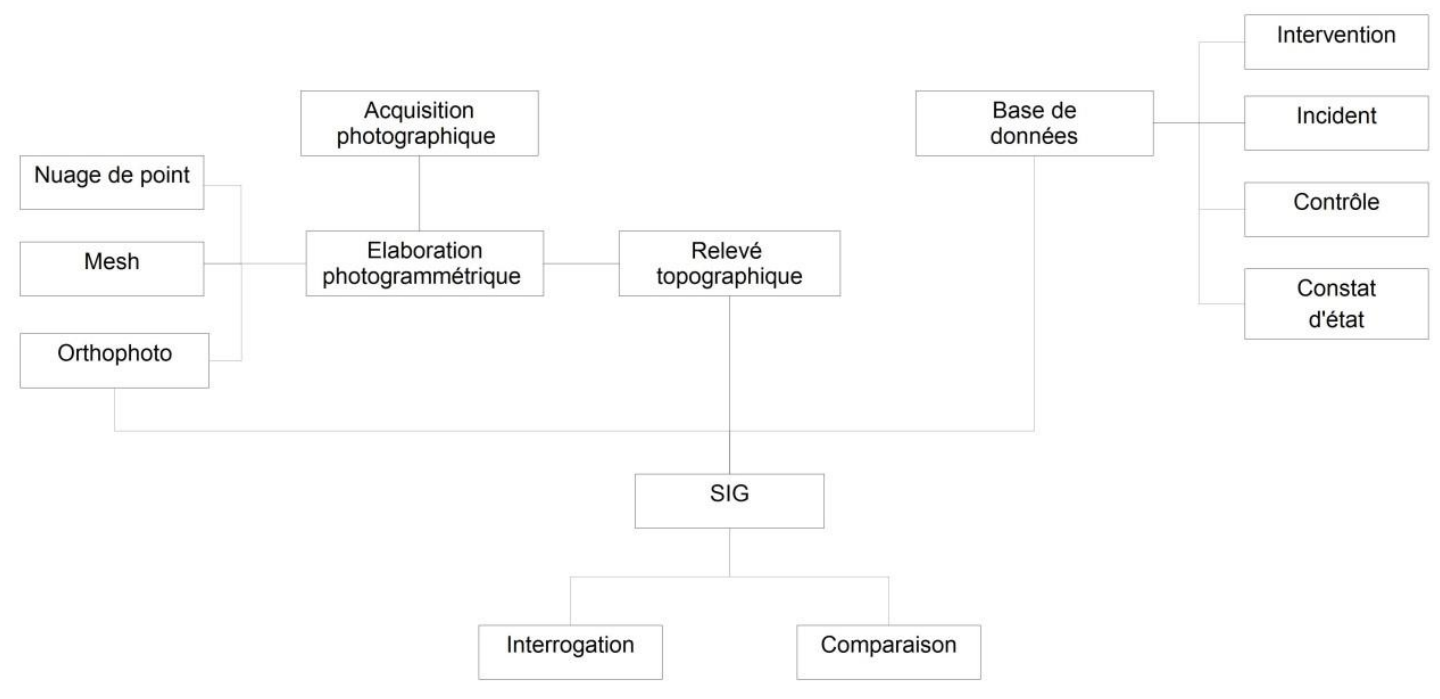

Figure 2. Structure opérationnelle du projet de suivi

\subsection{Le relevé topographique}

L'objectif était, de mettre au point un dispositif de surveillance nous permettant de suivre les variations physiques de l'épave au sein de son environnement muséographique. L'idée première était de considérer

Les outils de mesure topographique dont nous disposions (station Leica TS15P, précision angulaire 0.3 " (1mgon) ; précision des distances sans prisme $2 \mathrm{~mm}+2 \mathrm{ppm}$ ) et de vérifier s'ils étaient susceptibles de répondre aux besoins des conservateurs en charge du suivi du chaland.

Plusieurs contraintes matérielles sont à considérer dans la réalisation de nos travaux de mesures. En premier lieu, nous évoluons au sein d'un établissement à destination du public, les campagnes de levés ne peuvent donc s'effectuer que lorsque ce dernier est fermé aux visiteurs (le mardi). L'espace Muséographique dédié à l'exposition de la structure est tout en longueur, et comporte un grand nombre d'éléments architecturaux (colonnes, murs de refend, supports muséographiques). L'embarcation, est installée en contrebas de la zone de circulation, dans une fosse de $35 \mathrm{~m}$ de long, privilégiant un point de vue dominant sur les aménagements intérieurs. Cette fosse est elle-même entourée d'un garde corps métallique. Autant d'éléments masquant et obligeant à multiplier les points de stations afin d'effectuer l'ensemble des mesures nécessaires. Le contexte du musée rend impossible l'installation de stations topographiques pérennes au sol (repères de position au sol) sans détériorer ce dernier. Enfin, il est délicat de mettre en place des plaquettes de repérage ou des repères de position muraux avant d'avoir validé notre protocole de mesures.

Nous avons donc décidé de travailler dans un système de référence local et de positionner dans un premier temps un ensemble de 12 points de référence en hauteur afin d'éviter les détériorations accidentelles. Ces derniers sont matérialisés par des points rouges de $3 \mathrm{~mm}$ de diamètre comportant un évidement central. Ils sont destinés à être accessibles par des visées sans prisme. Ces points ont été consignés et servent de points d'appuis (figure 3). Le tachéomètre est positionné sur un trépied maintenu par une étoile assurant son maintien fixe sur la surface lisse du musée. Cinq à six mises en station sont 
nécessaires afin d'effectuer le tour de l'épave et de prendre les mesures les plus frontales possibles. Ces mises en station sont réalisées en utilisant la fonction "station libre », calées et calculées sur 3 à 5 points de référence et en faisant une moyenne selon les moindres carrés. Après chaque mise en station, ces mêmes points de référence utilisés sont mesurés et enregistrés pour vérification avant d'effectuer les relevés.

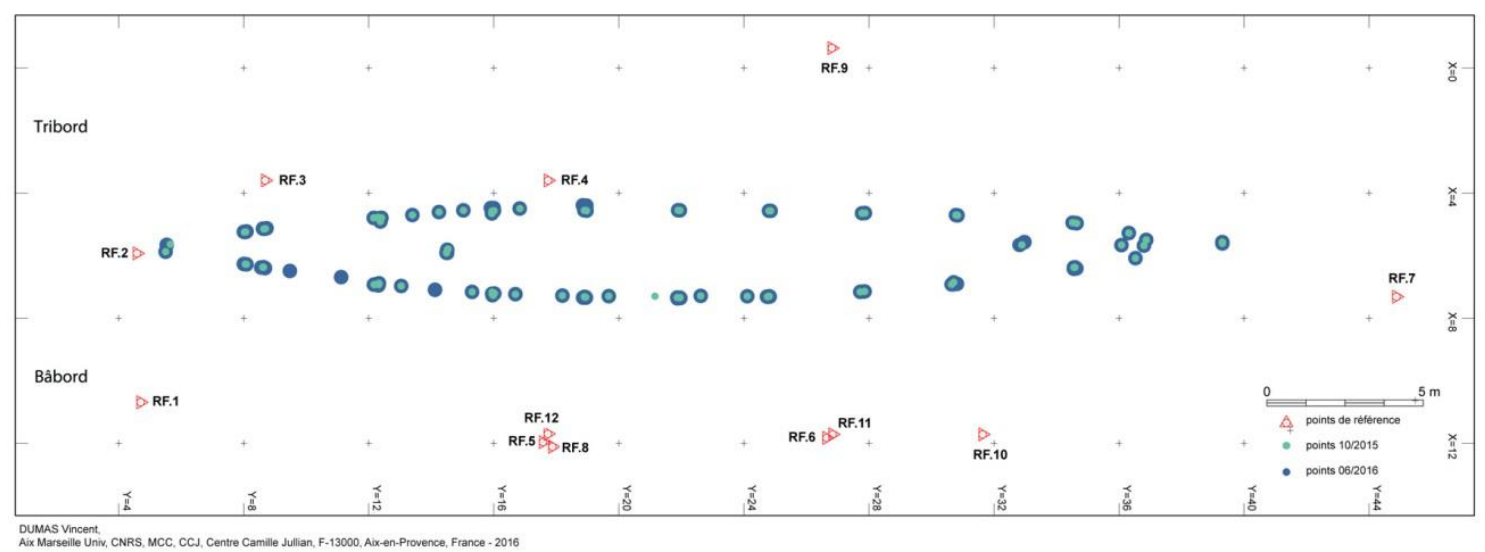

Figure 3. Le relevé topographique. Crédit : (O Vincent DUMAS (CCJ, CNRS).

Les points à relever sur la structure ont du être matérialisés à l'aide de micro pointe en inox entourées de petits points blancs (peinture blanche) de $3 \mathrm{~mm}$ de diamètre. Ces derniers sont invisibles pour les visiteurs mais suffisamment pour un œil averti. La micro pointe enfoncée au fil du bois permet une visée au réticule la plus précise possible. Enfin, la mesure s'effectue à l'aide du mode sans prisme du tachéomètre. C'est ainsi 77 points qui ont été mesurés en novembre 2015 et 118 lors de la seconde campagne en juin 2016. Cette augmentation des mesures est liée à la volonté de prendre en considération la structure en acier supportant le chaland. Les résultats obtenus, si l'on supprime certaines mesures aberrantes, nous offrent des variations moyennes millimétriques en $\mathrm{x}$ et sub-millimétriques en $\mathrm{Y}$ et en $\mathrm{Z}$. De prochaines campagnes sont prévues afin d'une part de valider ces résultats mais également de prendre de nouveaux points.

La précision de cette méthode de prise de mesures reste donc à conforter. Et il est peu probable que nous puissions garantir des résultats submillimétriques comme attendu. Qui plus est, le temps de chaque levé (3-4h), la nécessité d'être au moins deux opérateurs sur place ne permettent pas d'effectuer, rentablement, un suivi quotidien ni même hebdomadaire de l'évolution de la structure. D'autres pistes sont actuellement à l'étude. L'utilisation d'une machine portable de mesures de coordonnées (HandyProbe) détenue par la société Ipso Facto, déjà utilisée lors des relevés architecturaux du chaland, est à ce jour la solution la plus cohérente à mettre en place. En effet cette typologie d'instrumentation est dédiée aux opérations de métrologie dont l'inspection de pièces a des précisions submillimétriques recherchées par les conservateurs.

\subsection{Le relevé photogrammétrique et la base des données}

L'atelier de conservation-restauration du Musée d'Arles s'est tourné vers des moyens numériques pour tenter de rendre compte de manière quantitative des déformations déjà observées. Le constat des contraintes induites par la gestion d'un suivi papier et par la compilation d'observations empiriques, difficiles à relier dans une approche globale, ont en effet conduit le musée à envisager un nouvel outil de suivi, adapté au cas particulier d'AR3, mais éventuellement généralisable à d'autres épaves, pour des enjeux similaires. 
En 2015, la société Ipso Facto a réalisé un relevé 3D par photogrammétrie comme support scientifique (figure 4). Dans le cadre de la conservation, ce relevé permet de localiser et de mesurer les altérations de tous ordres, d'évaluer leurs évolutions et de situer les interventions, analyse et prélèvement.

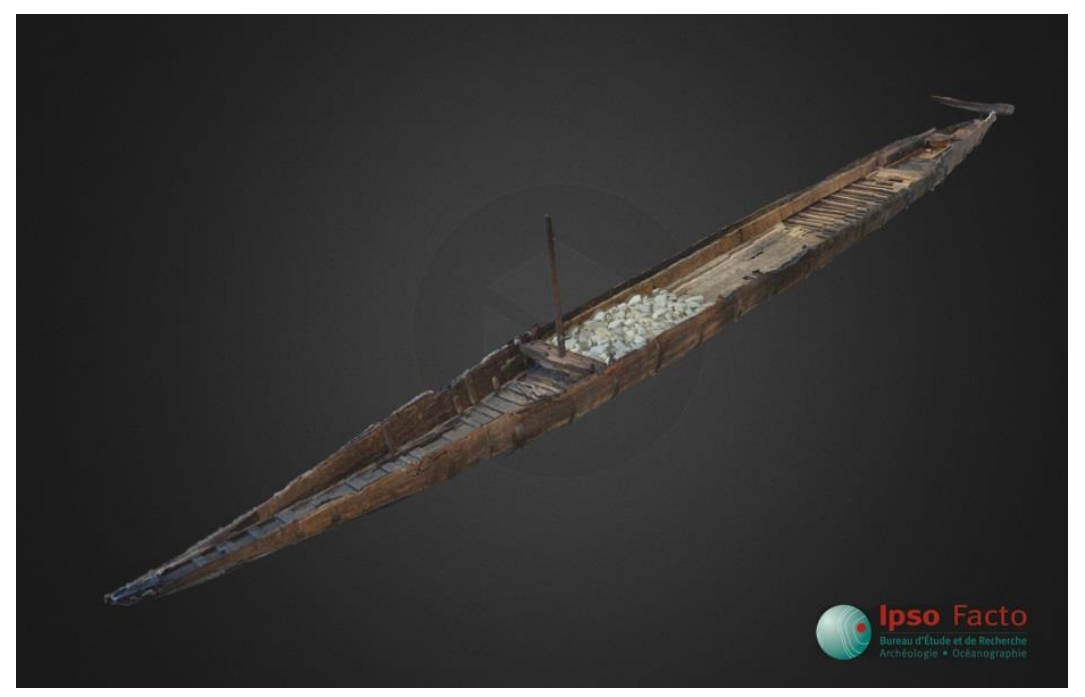

Figure 4. Le modèle photogrammétrique du chaland. Crédit : (O Daniela PELOSO (Ipso Facto).

La photogrammétrie, associée au relevé topographique, s'avère une technique appropriée pour rendre compte de l'évolution de l'épave en conservation. Cette méthodologie est très peu invasive, et, compte tenu des conditions spécifiques d'acquisition (dans un musée par exemple), fournit des données de grande précision et d'excellente qualité. Par ailleurs, sa mise œuvre relativement aisée, est à la portée d'un acteur de la conservation non spécialiste (un simple appareil photographique et une formation de quelques jours sont nécessaires).

Le relevé photogrammétrique du chaland a été réalisé avec l'acquisition de 2602 images haute définition capturées en trois jours. Le processus d'alignement des photos est établi sur la base de la position de l'appareil photo qui calcule l'information de profondeur de champs, pour combiner les images et produire un seul nuage de points dense.

L'élaboration a permis de générer un nuage total de 34 millions de points reconstruits. Le modèle final a été réduit à une véritable unité de mesure (mètres) en utilisant, comme référence, le plan du chaland. Toutes les images ont été alignées dans le même système de référence en appliquant les mêmes translation et mise à l'échelle, qui permettent de travailler dans le même système de référence.

Le modèle photogrammétrique a été également utilisé pour la production des ortho-images de chaque tronçon du bateau qui constituent le fond cartographique d'un système d'information ${ }^{4}$ conçu pour gérer et présenter tous les types de données spatiales (figure 5).

4 Le logiciel open-source utilisé est Qgis, A Free and Open Source Geographic Information System. http://www.qgis.org/en/site/ (C) 2017 ISTE OpenScience - Published by ISTE Ltd. London, UK - openscience.fr 


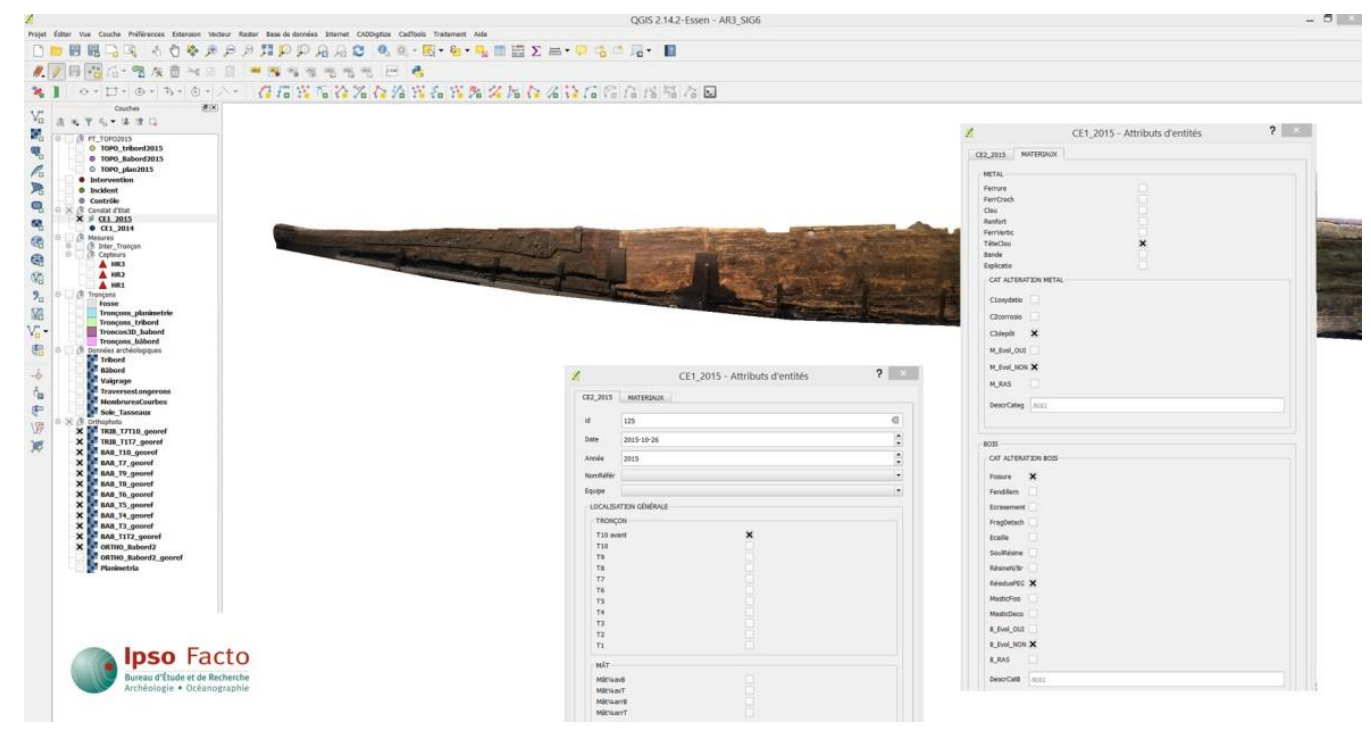

Figure 5. Les orthoimages et la base des données. Crédit : (O) Daniela PELOSO (Ipso Facto).

Depuis le premier constat réalisé en 2014 les observations sont consignées dans plusieurs dossiers. Dans une table attributaire figurent pour chacun des tronçons des données archéologiques, muséographiques sous forme de textes, de photos ou de relevés. On y retrouve également des informations chiffrées telles les distances entre les tronçons et des mesures de ph, d'humidité relative, ou le nombre de visiteurs. L'ensemble de ces données fourni des informations de nature très diverses qu'il est souhaitable de pouvoir interroger les unes par rapport aux autres. La base des données que nous avons construite en table attributaire, permet de collecter toutes ces informations.

Les renseignements de terrain et de traitement sont majoritairement exploitées par tronçons (10 tronçons numérotés $\mathrm{T} 1$ à $\mathrm{T} 10$ de la poupe vers la proue selon l'ordre du relevage); pour être logique les données relatives au suivi du bateau reprennent cette désignation. Nous avons donc une organisation spatiale par tronçon avec une localisation bâbord et tribord. Pour le suivi sanitaire les informations sont organisées selon la même localisation et concerne l'état de conservation et l'évolution du bois et du métal. La désignation des dégradations s'appuie sur l'élaboration d'un vocabulaire normatif. Les valeurs des mesures sont saisies directement et sont destinées à être comparées entre elles toujours selon le même système spatial.

L'objectif est de pouvoir analyser et interroger les données, pour constater une possible corrélation entre les réactions de l'épave face à leur environnement en fonction de leur nature intrinsèque, des traitements de conservation subis ou en cas d'accidents externes. Des correspondances sont remarquables aussi avec les facteurs environnementaux par rapport à la configuration de l'espace d'exposition.

Dans le contexte du suivi, le facile renouvellement des campagnes d'acquisition permet des comparaisons régulières avec les campagnes antérieures. Cette reproductibilité du protocole technique est très intéressante à souligner, dans la mesure où actuellement, dans les méthodes manuelles mises en œuvre, les acquisitions peu aisées induisent des limites de suivi.

Le modèle obtenu serait consultable en temps réel, sur un support portable (du type tablette numérique tactile). Le même support servirait également à l'enregistrement graphique (au moyen de critères et de figures simples et normées) des différents constats d'états hebdomadaires, annuels ou d'urgence, réalisés sur ou autour de l'épave. 


\section{Premiers résultats et conclusions}

Les deux relevés topographiques, réalisés par tachéométrie avec un intervalle de 7 mois (10 novembre 2015, 28 juin 2016) entre chacune des acquisition, nous ont permis d'assurer le monitorage du chaland.

Au niveau de chaque tronçon du chaland, différents point de contrôle ont été initialement fixés. Ils sont constitués par des cibles optiques sur le socle en fer, et par des marques physiques dans le bois, matérialisés par des petits clous à tête blanche.

L'étude expérimentale que nous menons a pour principal but la comparaison des résultats de mesures obtenues à partir de ces points fixes. Nous avons procédé comme suit :

- choix de 78 points aux limites de chaque tronçon,

- levée de 42 cibles optiques sur les supports latéraux des flancs, qui soutiennent le chaland et la pelle de gouvernail,

- acquisition de 12 points de référence hors épave dans la salle d'exposition du musée,

- collecte des données de mesure à partir des 120 points de contrôle fixés sur le chaland, et établissement des mouvements enregistrés et de leur évolution,

- traitement et analyse des résultats.

Pour faciliter l'interprétation, l'ensemble des résultats sera représenté sous forme graphique, reproduisant l'évolution des mouvements des points, durant deux années de relevés topographiques (figure 6).

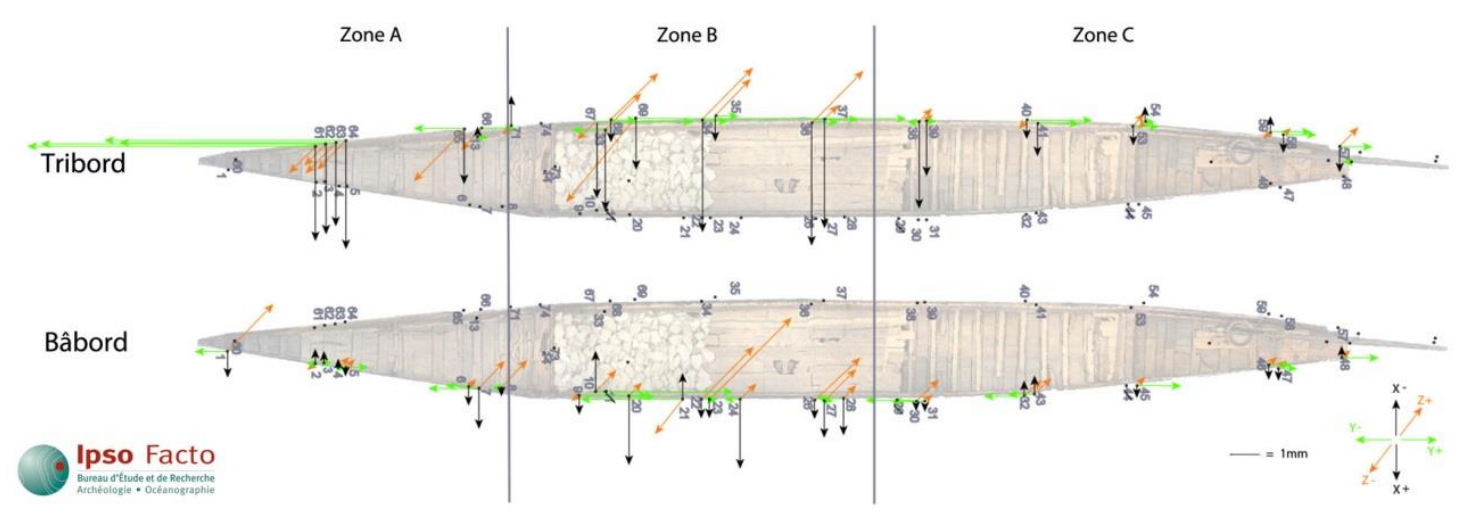

Figure 6. Représentation qui reproduit graphiquement les écarts relevés pour chaque point en 2015 et 2016, sur les flancs bâbord et tribord. Les écarts sont reproduits dans un système de coordonnées cartésiennes : en couleur noire, les écarts en $X$ (correspondant aux mouvements dans le sens de la largeur du chaland), en couleur verte, les écarts en $Y$ (correspondant aux mouvements dans le sens de la longueur), en couleurs orange, les écarts en $Z$ (correspondant aux mouvements en hauteur). Crédit : (O Daniela PELOSO, Mourad EL-AMOURI (Ipso Facto).

Les mesures que nous avons réalisées ont mis en évidence que trois parties distinctes du chaland semblent évoluer de façons différentes.

L'avant du bateau (zone A) présente les écarts plus remarquables, surtout à tribord. Nous avons enregistré des écarts :

- en X, de l'ordre de $2 \mathrm{~mm}$,

- en Y, de l'ordre de $6 \mathrm{~mm}$, 
- en Z, de l'ordre de $1 \mathrm{~mm}$.

Il semble opportun de rappeler que, du relevage à la restauration, l'avant du chaland a été soumis à un fort processus de remise en forme (à la découverte, les bordés des tronçons T9 et T10 étaient fortement déformés). Le protocole de monitorage mis en œuvre semble justement suggérer une instabilité constante à tribord pour les tronçons T9 et T10.

Pour la partie centrale du bateau (zone B), nous pouvons constater des mouvements constants sur les deux bordés :

- en X, de l'ordre de $1 \mathrm{~mm}$,

- en Y, de l'ordre de $1 \mathrm{~mm}$,

- en Z, de l'ordre de $1,5 \mathrm{~mm}$.

Ceux-ci correspondent sans doute aux efforts initialement imposés à cette partie du chaland, lors de son adaptation à la forme du socle (support métallique qui soutient l'épave pour la présenter dans l'exposition).

Pour la partie arrière du chaland (zone $\mathrm{C}$ ), les écarts pour chaque côté sont homogènes et de l'ordre de $0,5 \mathrm{~mm}$ dans les trois dimensions, cette partie de l'épave n'ayant pas subi d'importante contrainte lors de la mise en place, notamment parce qu'en partie reconstituée en ce qui concerne le flanc bâbord.

Pour contribuer significativement à l'étude du suivi de la conservation du chaland, il semble judicieux de comparer les relevés effectués en 2011 à la sortie de l'eau du chaland avec un appareil de mesure tridimensionnelle le C-Track 780 (en couleur bleu) au moment du relevage (donc avant le traitement de consolidation et de restauration, et la pose sur le socle), la modélisation proposée à l'époque de la mise en place des tronçons sur le socle (en couleur rouge) et le relevé photogrammétrique produit en 2015 après 2 ans d'exposition (en couleur verte).

Cette comparaison nous permet d'établir, de façon précise, l'évolution de la forme de chacun des tronçons. Ceci est illustré dans la figure 7 où sont présentées les trois superpositions pour les quatre relevés de coupe transversale au niveau des membrures M105, M112, M122, M134.
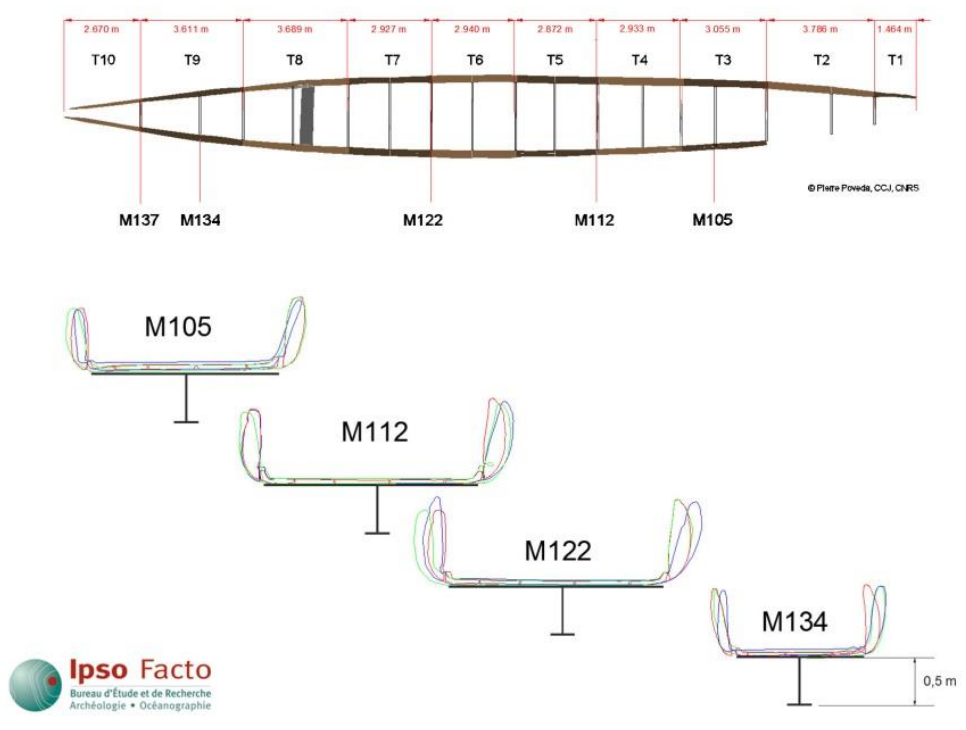

Figure 7. Comparaison entre les relevés effectués en 2011 (C-Track 780 avant les traitements), en 2013 (modélisation) et en 2015 (photogrammétrie). Crédit : (C Daniela PELOSO, Mourad EL-AMOURI (Ipso Facto). 
Dans la figure 8, et pour la seule membrure du tronçon T10, nous avons pu également faire apparaître un relevé supplémentaire, réalisé en 2013, après restauration par imprégnation à la résine PEG (en couleur magenta).

La comparaison permet de montrer les mouvements dans le temps, d'un tronçon qui a supporté nombreuses interventions ; encore une fois, le côté tribord semble montrer les variations plus importantes.
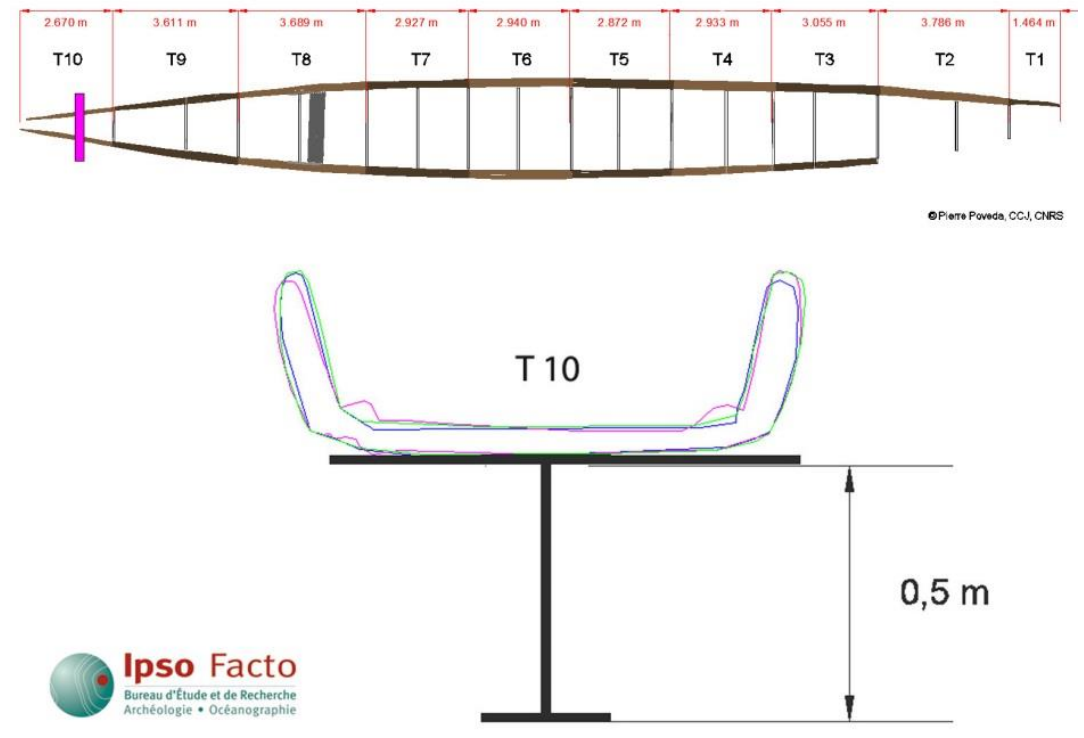

Figure 8. Tronçon T10. En couleur bleu, le relevé effectués en 2011 par C-Track 780 ; en couleur verte, le relevé photogrammétrique produit en 2015 ; en couleur magenta, le relevé fait en 2013 après restauration par imprégnation à la résine PEG. Crédit : (C) Daniela PELOSO, Mourad EL-AMOURI (Ipso Facto).

En conclusion, nous avons pu remarquer qu'effectivement un suivi précis des déformations du chaland était nécessaire pour assurer sa bonne conservation au sein d'une salle d'exposition. L'objectif à long terme est de pouvoir établir une corrélation chiffrée et cartographiée des interactions bateau-contexte d'exposition, aujourd'hui empiriquement constatées. Une fois utilisée pour d'autres épaves, le développement de cet outil sera un véritable atout pour leurs suivis, dans la mesure où la comparaison de conservations similaires sera rendue possible. Dans ce cadre, l'enregistrement numérique des informations sur un même logiciel permettrait l'obtention facilitée de données statistiques intéressantes.

Ce programme d'étude de l'état de conservation et des déformations des épaves, aujourd'hui soutenu par le Musée départemental d'Arles antique et la société Ipso Facto, le pourra être étendu à d'autres musées ou chercheurs intéressés.

\section{Bibliographie}

Bernard-Maugiron, H., L. Blanc and P. de Viviés, A Case Study: The analysis and restoration of pyrite-contaminated composite waterlogged wood of a Gallo-Roman barge from Arles. In Proceedings of the 12th ICOM-CC Group on Wet Organic Archaeological Materials Conference, Istanbul, 2013

Bernard-Maugiron, H, M.-L. Courboulès, 2016, "Is it possible and reasonable to treat in 2 years a 31 meter long waterlogged roman boat?" and "Development of a monitoring protocol for preservation of Arles Rhône 3 wreck ». In Proceedings of the 13th ICOM-CC Group on Wet Organic Archaeological Materials Conference, Firenze.

Caillat, L., L. Meunier-Salinas and M-A Coignard. 2015, Régénération continue des bains de PEG utilisés pour la consolidation des bois archéologiques gorgés d'eau. TECHNE ${ }^{\circ} 42: 115-120$. 
Colson A., Guery J., Ditta M., 2015, “Bremen Cog” - long term monitoring of deformation processes, in Condition.2015 Conservation and Digitalization, Conference Proceedings, Gdansk, pp.107-111

Jacobson J., 2003, "Measuring the Vasa." , [in:] Proceedings from the International Conference on Maritime Heritage, Wessex 2003, pp.183-190.

Marlier, S. 2014, Arles-Rhône 3, un Chaland Gallo-Romain du 1er Siècle après Jésus-Christ, Archaeonautica 18, CNRS éditions, Paris

Sintes, C., S. Marlier., and H. Bernard-Maugiron, 2013, "L'opération Arles-Rhône 3" Du fleuve au musée, National Geographic, édition spéciale pour le musée de l'Arles antique : 48-65.

Wessex, 2003, Deformationsmätningar av skeppet Vasa. In SINUS, Number 3, pp 5 -8. 